\title{
Sept approches complémentaires pour répondre à une question fondamentale
}

Daniel Favre

\section{OpenEdition}

\section{Journals}

Édition électronique

URL : http://journals.openedition.org/trema/347

DOI : 10.4000/trema.347

ISSN : 2107-0997

\section{Éditeur}

Faculté d'Éducation de l'université de Montpellier

\section{Édition imprimée}

Date de publication : 1 mars 2007

Pagination : 1-3

ISSN : 1167-315X

\section{Référence électronique}

Daniel Favre, «Sept approches complémentaires pour répondre à une question fondamentale », Tréma [En ligne], 27 | 2007, mis en ligne le 12 janvier 2010, consulté le 22 septembre 2020. URL : http:// journals.openedition.org/trema/347 ; DOI : https://doi.org/10.4000/trema.347

Ce document a été généré automatiquement le 22 septembre 2020.

Trema 


\title{
Sept approches complémentaires pour répondre à une question fondamentale
}

\author{
Daniel Favre
}

1 L'école semble avoir toujours été un lieu où savoir et autorité se présentent comme indissociables et s'engendrent mutuellement.

2 Ce modèle d'école traditionnel paraît s'appliquer à une grande partie de l'Antiquité où les connaissances issues de la Mésopotamie et de l'Égypte étaient jalousement gardées, conservées pour être fidèlement transmises à des élèves «muets » qui, eux - mêmes, deviendront à leur tour des maîtres. Mais c'est aussi au VIe siècle avant J.C., donc en pleine Antiquité, que la science et la philosophie vont constituer une rupture par rapport à ces traditions et aux écoles qui les servaient.

3 «En l'espace de moins de trois siècles, le monde grec a connu l'invention de l'alphabet phonétique, de la monnaie et de la démocratie. Ces trois événements, bien qu'ils aient chacun leur explication historique propre, s'articulent avec la naissance de la science et de la philosophie» (PICHOT, 1991)1.

4 Selon l'épistémologue Karl POPPER, il aurait bien existé, d'après les textes postérieurs à cette époque qui nous sont parvenus, un moment fondateur avant Socrate dans l'école d'ELÉE et l'école Ionienne. Ce moment se serait produit lorsque ANAXIMANDRE (- 610 547 ?), élève ou interlocuteur privilégié de THALÈS (- 624 - 546 ?), commença à critiquer les propos de THALÈS et lorsque celui - ci l'encouragea à poursuivre. En l'encourageant au lieu de le rejeter, THALÈS, en tant que représentant de l'autorité, légitima en quelque sorte un nouveau comportement cognitif : la recherche des contre - évidences, i.e. l'esprit critique.

5 Une liberté nouvelle était née ainsi qu'un nouveau mode de rapport avec le savoir et l'autorité; à la suite d'ANAXIMANDRE, les disciples milésiens vont presque tous critiquer leurs maîtres, ce qui ne sera pas forcément le cas dans les autres écoles grecques. 
6 En trouvant « intéressant » le discours qui critique, donc qui « dé - construit », THALÈS initie une rupture avec une autre valeur qui visait à conserver les connaissances comme des trésors et à les conserver telles quelles pour les transmettre aux générations futures. Le discours qui déconstruit sera à partir de ce moment - là valorisé car il permet à la pensée de progresser. Le progrès vient de la critique qui permet de mieux préciser le domaine de validité de l'activité de penser.

7 La Philosophie et la Science trouvent dans ce changement de valeur leur origine. Ce changement engendre un nouveau mode de rapport au savoir produit par l'adoption de l'attitude critique du savoir et un nouveau mode de rapport à l'autorité puisque celle - ci ne s'assimile plus à l'accumulation des connaissances par une personne.

8 La suite de notre histoire montrera que la science et la philosophie auront des difficultés à émerger socialement. Jusqu'aux trois révolutions européennes du XVIIe siècle, ce ne sera pas facile, voire dangereux, de penser différemment de la norme et c'est encore le cas aujourd'hui dans certains pays. Ainsi, pendant vingt siècles la philosophie et la science vont rester confondues et leur usage très peu généralisé jusqu'à la renaissance en Occident. Bien au contraire les "grands prêtres » du savoir vont établir leur pouvoir en conservant et en thésaurisant les connaissances et en punissant tous ceux qui voulaient les remettre en question.

9 Entre l'autorité qui ne vise qu'à s'asseoir sur des connaissances immuables et à se reproduire à l'identique en écartant par tous les moyens ce qui pourrait la déstabiliser et l'autorité qui vise à rendre auteur, à faire advenir un sujet, qui accueille la personne et la critique, il y a un monde et pourtant on emploie le même mot.

10 Tenter d'approcher le phénomène complexe de l'autorité, de distinguer des postures qui assujettissent et dont la finalité est la soumission de celui sur qui elles s'exercent, de celles qui posent un cadre, un contenant pour permettre à un être en devenir de grandir sera l'objectif principal des chapitres qui vont suivre.

11 L'histoire récente de notre civilisation montre en effet que l'impulsion donnée par THALÈS de MILET n'a pas encore transformé l'autorité des maîtres, pas plus que la démarche scientifique n'a été adoptée de manière unanime par tous ceux qui exercent l'autorité. Ce serait d'ailleurs intéressant de vérifier si la majorité des enseignants actuels préfèrent les ANAXIMANDRE ou les élèves qui se conforment.

12 Pourtant l'évolution de nos sociétés semble imposer en Europe de nouvelles missions aux enseignants : former des citoyens et transmettre des savoirs où il est bien clair que c'est la transmission des savoirs qui doit être mise au service de la première mission ${ }^{2}$.

13 Il s'agit donc de développer l'esprit critique des élèves et d'éduquer à la responsabilité. Dans ce cas, si les anciennes formes de l'autorité ont perduré jusqu'à nos jours, le besoin de soumettre, qui anime ceux qui les servent, ne constitue - $t$ - il pas un obstacle?

14 Le problème actuel de l'École est - il donc de restaurer son autorité ou d'instaurer une autorité nouvelle?

15 Pour tenter de répondre à ces questions Ion ABULESCU montre comment, en Roumanie, les valeurs associées à la démocratie et à l'éducation à la citoyenneté bouleversent les traditions et les formes de l'autorité. La résistance de ces différentes formes au changement appelle un diagnostic dont va dépendre la mise en actes ou non des relations de coopération entre les élèves, comme le montre une étude faite au Québec par Jean - Claude KALUBI, Sylvie HOUDE et Catherine GARNIER. En France, Gérard 
PITHON et Xavier BARRANDON mettent en évidence d'importantes divergences entre les représentations de la sanction des élèves et celles des enseignants, ce qui peut constituer un obstacle pour que l'autorité de ces derniers puisse être acceptée. Or, sans cette acceptation, sans le cadre et les limites qu'apporte ce modèle d'adulte qu'est l'enseignant, Dominique GINET montre que le sujet ne peut pas grandir et s'émanciper. Cela demande une grande vigilance de la part de l'enseignant d'être dans la posture de celui ou de celle qui accepte l'altérité et c'est tout l'intérêt de l'approche clinique de Jacques CABASSUT, de repérer les difficultés que peut recéler la relation d'autorité avec des autres qui, quelquefois, nous paraissent tellement étrangers. La dernière contribution, celle de Daniel FAVRE, Allen LARÈS et Marie - Louise MARTINEZ, souligne comment la peur est souvent au rendez - vous pour celui qui, exerçant une autorité de domination, est dépendant des réactions de soumission des autres et comment, à 20 ans d'intervalle, les jeunes attendent toujours autre chose de l'autorité de l'adulte, un cahier des charges exaltant, mais qui peut conduire à des renoncements nécessaires.

\section{NOTES}

1. PICHOT A. (1991) La naissance de la science T.2, coll. Folio - essais, Gallimard, Paris.

2. CORNU, B \& BRIHAULT, J. (2001) Pour une rénovation du dispositif de formation des enseignants. Rapport à Monsieur le Ministre de l'Éducation Nationale du 5 janvier.

\section{AUTEUR}

\section{DANIEL FAVRE}

Maître de conférences, Professeur des Universités en Sciences de l'Éducation IUFM de Montpellier 